\title{
Limited cross-reactivity of mouse monoclonal antibodies against Dengue virus capsid protein among four serotypes
}

This article was published in the following Dove Press journal:

Biologics:Targets and Therapy

22 November 2012

Number of times this article has been viewed

\author{
Megumi Noda' \\ Promsin Masrinoul' \\ Chaweewan Punkum' \\ Chonlatip Pipattanaboon ${ }^{2,3}$ \\ Pongrama Ramasoota ${ }^{2,4}$ \\ Chayanee Setthapramote ${ }^{2,3}$ \\ Tadahiro Sasaki ${ }^{6}$ \\ Mikiko Sasayama' \\ Akifumi Yamashita ${ }^{1,5}$ \\ Takeshi Kurosu ${ }^{6}$ \\ Kazuyoshi Ikuta ${ }^{6}$ \\ Tamaki Okabayashi' \\ 'Mahidol-Osaka Center for Infectious \\ Diseases, ${ }^{2}$ Center of Excellence for \\ Antibody Research, ${ }^{3}$ Department \\ of Microbiology and Immunology, \\ ${ }^{4}$ Department of Social and \\ Environmental Medicine, Faculty \\ of Tropical Medicine, Mahidol \\ University, Ratchathewi, Bangkok, \\ Thailand; ${ }^{5} \mathrm{Graduate}$ School of Life \\ Science, Tohoku University, Sendai, \\ Miyagi, ${ }^{6}$ Department of Virology, \\ Research Institute for Microbial \\ Diseases, Osaka University, Suita, \\ Osaka, Japan
}

Correspondence:Tamaki Okabayashi Mahidol-Osaka Center for Infectious Diseases, 420/6 Ratchathewi Rd, Ratchathewi, Bangkok 10400,

Thailand

Tel +66 23549156

Fax +66 23549157

Email tamaoka@biken.osaka-u.ac.jp
Background: Dengue illness is one of the important mosquito-borne viral diseases in tropical and subtropical regions. Four serotypes of dengue virus (DENV-1, DENV-2, DENV-3, and DENV-4) are classified in the Flavivirus genus of the family Flaviviridae. We prepared monoclonal antibodies against DENV capsid protein from mice immunized with DENV-2 and determined the cross-reactivity with each serotype of DENV and Japanese encephalitis virus.

Methods and results: To clarify the relationship between the cross-reactivity of monoclonal antibodies and the diversity of these viruses, we examined the situations of flaviviruses by analyses of phylogenetic trees. Among a total of 60 prepared monoclonal antibodies specific for DENV, five monoclonal antibodies stained the nuclei of infected cells and were found to be specific to the capsid protein. Three were specific to DENV-2, while the other two were cross-reactive with DENV-2 and DENV-4. No monoclonal antibodies were cross-reactive with all four serotypes. Phylogenetic analysis of DENV amino acid sequences of the capsid protein revealed that DENV-2 and DENV-4 were clustered in the same branch, while DENV-1 and DENV-3 were clustered in the other branch. However, these classifications of the capsid protein were different from those of the envelope and nonstructural 1 proteins. Phylogenetic distances between the four serotypes of DENV were as different as those of other flaviviruses, such as Japanese encephalitis virus and West Nile virus. Large variations in the DENV serotypes were comparable with the differences between species of flavivirus. Furthermore, the diversity of flavivirus capsid protein was much greater than that of envelope and nonstructural 1 proteins. Conclusion: In this study, we produced specific monoclonal antibodies that can be used to detect DENV-2 capsid protein, but not a cross-reactive one with all serotypes of DENV capsid protein. The high diversity of the DENV capsid protein sequence by phylogenetic analysis supported the low cross-reactivity of monoclonal antibodies against DENV capsid protein.

Keywords: Dengue virus, capsid protein, monoclonal antibody, cross-reactivity

\section{Introduction}

Dengue illness, such as dengue fever and dengue hemorrhagic fever/dengue shock syndrome, is one of the important mosquito-borne viral diseases in tropical and subtropical regions. ${ }^{1}$ Four serotypes of dengue virus (DENV-1 to DENV-4) are classified in the genus Flavivirus of the family Flaviviridae. This also includes Japanese encephalitis virus, West Nile virus, and yellow fever virus. ${ }^{2}$

The $11 \mathrm{~kb}$ DENV genome is translated into a single polyprotein, which is subsequently processed by proteases into structural and nonstructural proteins. Three structural proteins, i.e. capsid, premembrane/membrane, and envelope, make up the virus particle. ${ }^{3}$ The DENV capsid protein is a relatively small, highly positively 
charged $12 \mathrm{kDa}$ protein and an essential factor during virion assembly. ${ }^{4,5}$ Interestingly, DENV capsid protein is found in the nucleus and nucleoli of infected cells as early as 6 hours after infection, well before formation of infectious virus. ${ }^{6,7}$ Previous reports have suggested nucleolar accumulation of DENV capsid protein may result from its interaction with RNA or nucleoli proteins related to regulation of ribosome synthesis, mRNA processing, and DNA replication. ${ }^{5,8,9}$ This nuclear localization of capsid protein appears to be conserved among flaviviruses, including West Nile virus, Japanese encephalitis virus, Kunjin virus, and hepatitis $C$ virus. ${ }^{10-13}$

In general, antibodies against the capsid proteins of viral particles, such as human immunodeficiency virus (HIV) Gag protein and influenza virus nucleoprotein, have been used in antigen-capture diagnosis kits ${ }^{14,15}$ because of their wide range of cross-reactivity, even with other subtypes, compared with that of the envelope proteins. ${ }^{16,17}$ Similar approaches using antigen-capture diagnostic or enzyme-linked immunoassay kits have been used for other viruses, such as Rift Valley fever virus, ${ }^{18}$ hepatitis B virus, ${ }^{19}$ hepatitis C virus, ${ }^{20}$ and Ebola virus. ${ }^{21}$ Such viral structural capsid proteins are located inside, not on the surface, of viral particles and the amount of internal protein exists at a higher concentration than that of surface protein. Therefore, use of monoclonal antibodies against the internal structural protein could have advantages for developing rapid diagnostic kits. In addition, expression of DENV capsid protein has been detected at a very early stage in infected mammalian cells. ${ }^{33}$ Thus, if antibodies against DENV capsid protein were also widely cross-reactive among all four serotypes of DENV, like those against HIV Gag protein and influenza virus nucleoprotein, monoclonal antibodies recognizing common antigenic regions on DENV capsid proteins for all four DENV serotypes could be highly useful for developing a rapid diagnostic test kit widely cross-reactive with all four serotypes in the acute phase. However, monoclonal antibodies to the capsid protein has not been used for development of a diagnostic kit to detect this viral infection, and monoclonal antibodies to DENV nonstructural 1 protein have been used instead for developing diagnostic kits. 22,23

In this study, we attempted to produce monoclonal antibodies against DENV capsid protein that could be crossreactive with all four serotypes of DENV. By immunizing BALB/c mice with DENV-2, we obtained a total of five hybridoma clones producing specific antibodies against DENV capsid protein. We hypothesized that a monoclonal antibody against DENV capsid protein would recognize common antigenic regions on the capsid proteins of all four DENV serotypes and be very useful for developing a diagnostic test kit widely cross-reactive with all four serotypes. The purpose of this study was to clarify the relationship between the cross-reactivity of monoclonal antibodies and the diversity of these viruses, and we examined the situation of flaviviruses by analysis of phylogenetic trees.

\section{Materials and methods Cells and viruses}

Vero cells (from an African green monkey kidney epithelial cell line) and B-7 cells (from a BALB/c mouse cell line) ${ }^{24}$ were maintained in Eagle's Minimum Essential Medium (MEM, HyClone Laboratories Inc, Logan, UT) supplemented with $10 \%$ fetal calf serum (HyClone Laboratories Inc). PAI cells (from a mouse myeloma cell line) were cultured in Roswell Park Memorial Institute 1640 (RPMI 1640, HyClone Laboratories Inc) medium supplemented with $10 \%$ fetal calf serum. All cell lines were cultured at $37^{\circ} \mathrm{C}$ in a $5 \% \mathrm{CO}_{2}$ incubator. C6/36 cells (from an Aedes albopictus cell line) were grown in Leibovitz-15 medium (HyClone Laboratories, Inc) supplemented with $0.3 \%$ tryptose phosphate broth and $10 \%$ fetal calf serum at $28^{\circ} \mathrm{C}$.

Viral stocks of DENV-1 (Mochizuki strain), DENV-2 (New Guinea C strain), DENV-3 (H87 strain), and DENV-4 (H241 strain) were prepared as culture fluids from C6/36 cells infected with the individual serotypes and cultured for 7-9 days. In addition, the Nakayama strain of Japanese encephalitis virus was also similarly cultured in C6/36, and the culture fluid was used as a virus stock. Infectivity titers of these viruses were determined by the number of focusforming units, as described previously. ${ }^{25}$

\section{Mouse immunization and monoclonal antibody preparation}

For preparation of DENV-2 antigens, we used two types of antigens, ie, B7-cells infected with DENV-2 and 50\% brain homogenate with phosphate-buffered solution (-) from suckling BALB/c mice that were injected with DENV-2. B7-cells infected with DENV-2, at a multiplicity of infection of 0.1 and cultured for 2 days, were harvested by scraping and precipitated by centrifugation at $1000 \mathrm{rpm}$ for 5 minutes. Brain homogenate was prepared by intracerebral injection of DENV-2 in suckling mice. As soon as the injected mice showed symptoms, their brains were collected and frozen at $-80^{\circ} \mathrm{C}$ until use. Antigens were kept at $-80^{\circ} \mathrm{C}$ until use for immunization of mice. Three 4-week-old female BALB/c mice (National Laboratory Animal Center, Mahidol University, Bangkok, Thailand) were immunized with $2.5 \times 10^{6}$ infected cells or $300 \mu \mathrm{L}$ of homogenized brain 
mixed with complete Freund's adjuvant (Sigma-Aldrich, Saint Louis, MO), as described previously. ${ }^{26}$ Each mouse was injected intraperitoneally with $300 \mu \mathrm{L}$ of mixed antigen. Immunized mice were intraperitoneally boost-immunized 3-4 times with similarly prepared antigens without adjuvant. This study was approved by the Faculty of Tropical Medicine Animal Care and Use Committee, Mahidol University, Bangkok, Thailand (FTM-ACUC 2011/003). Three days after the final booster immunization, splenocytes were prepared and subjected to fusion with PAI cells using polyethylene glycol 1500 (Roche Diagnostic Corporation, Basel, Switzerland). Fused cells were cultured in Dulbecco's Modified Eagle's Medium supplemented with 15\% fetal calf serum and hypoxanthine-aminopterin-thymidine (Gibco, Grand Island, NY). Monoclonal antibodies produced from hybridomas were screened with DENV-2-infected Vero cells by an immunofluorescence assay. Hybridomas were cloned twice by limiting dilutions using 96-well microplates.

\section{Expression of DENV-2 capsid protein}

The DENV-2 capsid protein expression plasmid, pCAGGSPM2 FLAG-DEN2 core 100-HA, was kindly provided by $\mathrm{Y}$ Matsuura at the Research Institute for Microbial Diseases of Osaka University, Suita, Osaka, Japan. Expression of this plasmid was confirmed by Western blotting and immunofluorescence assays using an anti-Flag M2 monoclonal antibody (Sigma-Aldrich). The plasmid vector was transfected by Lipofectamine 2000 (Invitrogen, Carlsbad, CA) for Vero cells.

\section{Immunofluorescence assay}

Vero cells were seeded into 96-well plates for preparation of DENV antigens in infected cells and DENV-2 capsid protein in transfected cells. After incubation for 16-24 hours, they were infected with each serotype of DENV or transfected with pCAGGS-PM2 FLAG-DEN2 core 100-HA plasmid. Two days after infection or transfection, the cells were fixed with $4 \%$ paraformaldehyde in phosphate-buffered solution for 30 minutes at room temperature. Vero cells infected with Japanese encephalitis virus were also similarly prepared. The fixed cells were permeabilized with $1 \%$ Triton $\mathrm{X}-100$ in phosphate-buffered solution for 5 minutes at room temperature, and then incubated with hybridoma culture fluid for one hour. They were then washed three times with phosphate-buffered solution and further treated with Alexa Fluor $^{\circledR} 488$ goat anti-mouse $\operatorname{IgG}$ antibody (Invitrogen) at a dilution of 1:500 for 45 minutes. Finally, they were washed three times with phosphate-buffered solution prior to observation by fluorescence microscopy (IX71, Olympus, Tokyo, Japan).

\section{Western blotting assay}

DENV-infected Vero cells were dissolved in sodium dodecyl sulfate-polyacrylamide gel electrophoresis (SDS-PAGE) sample buffer with beta-mercaptoethanol and heated at $100^{\circ} \mathrm{C}$ for 5 minutes. The samples were separated in $12 \%$ SDS-PAGE gel and transferred to a polyvinylidene fluoride membrane (Millipore Corporation, Bedford, MA). The membrane was incubated for 12 hours with antibody produced by the hybridoma clones and then with horseradish peroxidase-conjugated anti-mouse IgG (KPL, Washington, DC) for one hour. The reactive viral protein was visualized using an ECL WB detection agent (GE Healthcare, Buckinghamshire, UK).

\section{Phylogenetic analysis of capsid, envelope, and nonstructural I proteins}

All available sequences of DENV-1 to DENV-4, Japanese encephalitis virus, and West Nile virus were downloaded from the National Center for Biotechnology Information website (http://www.ncbi.nlm.nih.gov/protein) on February 7 , 2012. The capsid, envelope, and nonstructural 1 amino acid sequences were extracted from these sequences using the results of a BLAST (Basic Local Alignment Search Tool) homology search ${ }^{27}$ against the corresponding proteins. These sequences were aligned using MAFFT version $6.705 b^{28}$ after removing redundant sequences. Phylogenetic trees were constructed using the neighbor-joining method ${ }^{29}$ with MEGA5. ${ }^{30}$ All positions containing gaps and missing data were eliminated.

\section{Results \\ Preparation of mouse monoclonal antibodies against DENV}

To prepare hybridoma clones producing anti-DENV monoclonal antibodies, spleen cells derived from mice immunized with DENV-2 infected cells were fused with myeloma cell lines as previously described. ${ }^{26}$ After screening and single-cell cloning, we obtained a total of 60 hybridoma clones specifically reacted with DENV-2-infected Vero cells, but not with uninfected Vero cells, by indirect immunofluorescence assay. The 4G2 monoclonal antibody, available as an anti-flavivirus, ${ }^{31}$ was used as a positive control for the immunofluorescence assay. This $4 \mathrm{G} 2$ reacted with the envelope protein of DENV in the cytoplasm of infected Vero cells (Figure 1). Of the 60 clones generated in this study, five clones were reacted 


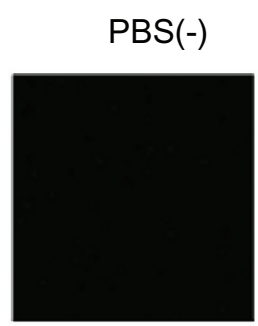

C2

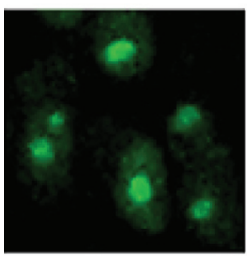

$5 \mathrm{~F} 12$
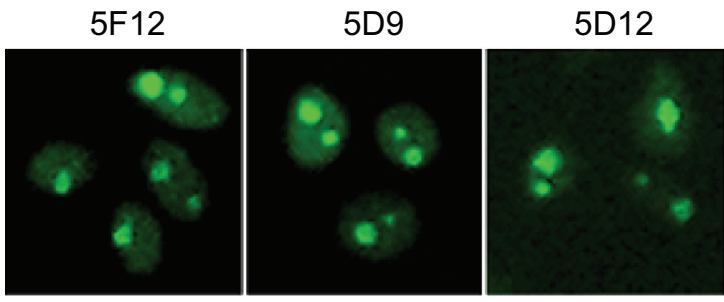

Figure I Indirect immunofluorescence assay of anti-DENV-2 reacted with the nuclear protein of DENV-2 infected cells.

Notes: Vero cells were infected with DENV-2 and fixed 48 hours after infection with $4 \%$ paraformaldehyde. Cells were stained with the indicated hybridoma culture fluid, C2, C99, 5FI2, 5D9 and 5DI2, or 4G2 (anti-envelope monoclonal antibody) as a control.

with the nuclei of infected cells (Figure 1). Two clones (C2 and C99) were derived from mice immunized with the brain homogenate of BALB/c suckling mice injected with DENV-2, while three clones (5F12, 5D9, and 5D12) were derived from three mice immunized with B7-cells infected with DENV2. All the other 55 monoclonal antibodies reacted with the cytoplasm of infected cells (data not shown).

\section{Monoclonal antibodies recognizing DENV capsid protein}

To identify a viral protein recognized by five monoclonal antibodies which were shown to stain the nuclei of infected cells as above, a Western blot assay with the culture supernatants of individual hybridoma clones was carried out using the lysate of DENV-2-infected Vero cells that were treated with betamercaptoethanol and heated at $100^{\circ} \mathrm{C}$. The $\mathrm{C} 2$ monoclonal antibody reacted specifically with $12 \mathrm{kDa}$ DENV-2 capsid protein (Figure 2A). To confirm that this binding protein was the capsid protein, Vero cells were transfected with plasmid expressing recombinant DENV-2 capsid protein. This monoclonal antibody reacted with recombinant DENV-2 capsid protein in transfected cells on Western blotting and immunofluorescence assay (Figure 2 and Table 1). Although the other four monoclonal antibodies (C99, 5F12, 5D9, and 5D12) did not react with the $12 \mathrm{kDa}$ capsid protein on Western blot assay (Figure 2A), these monoclonal antibodies similarly reacted with recombinant capsid protein in transfected Vero cells by immunofluorescence assay (Figure 2B). Thus, we concluded that these five monoclonal antibodies recognized the DENV capsid protein.

\section{Cross-reactivity of monoclonal antibodies to DENV capsid protein}

To determine the cross-reactivity of the five above-mentioned monoclonal antibodies to DENV capsid protein with each serotype of DENV and Japanese encephalitis virus, individual monoclonal antibodies in culture supernatants were examined by immunofluorescence assay using Vero cells infected with DENV-1 to DENV-4, as well as Japanese encephalitis virus (Figure 3). The cross-reactivities of our five monoclonal antibodies are summarized in Table 1. Three monoclonal antibodies, C2, 5F12, and 5D9, were reacted specifically with only DENV-2 but not with the other serotypes, whereas the other two monoclonal antibodies, C99 and 5D12, were cross-reacted with DNEV-2 and DENV-4. None of these five monoclonal antibodies showed cross-reactivity with DENV-1 or DENV-3. In addition, there were no positive reactions with these five monoclonal antibodies in Vero cells infected with Japanese encephalitis virus (Figure 3 and Table 1).

\section{Phylogenetic analysis of flavivirus}

Examination of anti-DENV capsid protein monoclonal antibodies for their cross-reactivity among four serotypes of DENV, as well as Japanese encephalitis virus and West Nile virus belonging to the same flavivirus, revealed only limited cross-reactivity. Therefore, we examined the situations for individual serotypes of DENV as well as Japanese encephalitis virus and West Nile virus by analysis of phylogenetic trees. The sequences of flavivirus capsid, envelope, and nonstructural 1 proteins all derived from the National Center For Biotechnology Information database were used for construction of individual phylogenetic trees. The numbers of the amino acid sequences used for phylogenetic analysis were shown in Table 2.

As observed in the cross-reactivity of monoclonal antibodies, phylogenetic analysis of the capsid protein showed that DENV-2 and DENV-4 were clustered in the same branch, while DENV-1 and DENV-3 were clustered in the other branch (Figure 4A, capsid protein). This meant that the capsid protein of DENV-2 was most closely related 


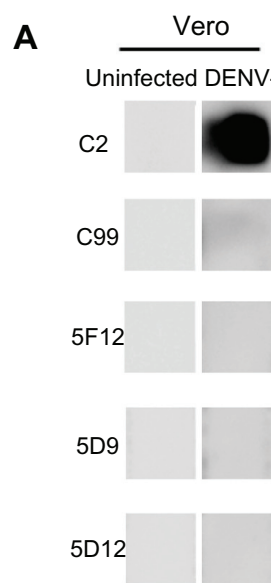

Anti-Flag
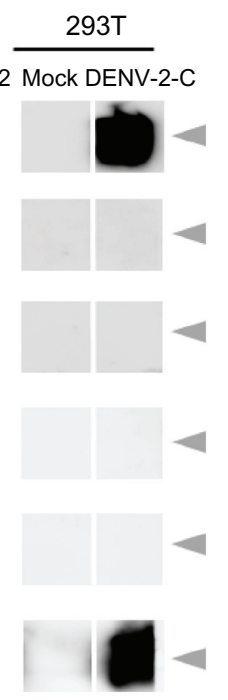

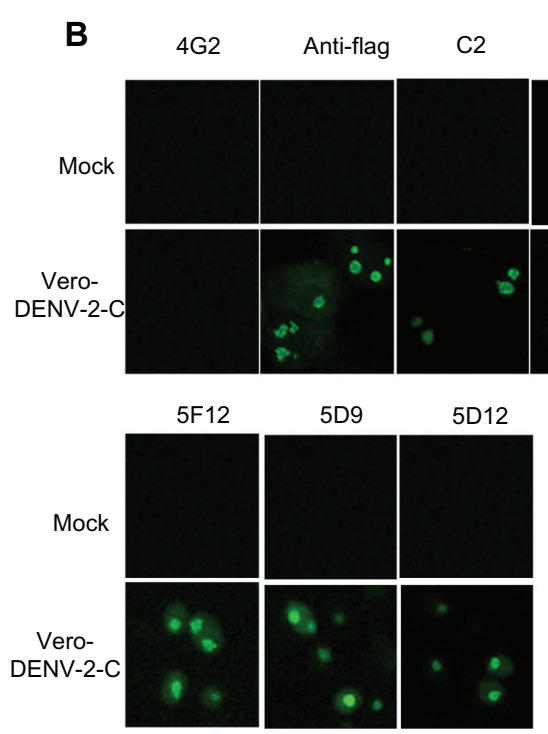

Figure 2 Identification of antigenic DENV-2 capsid protein. (A) Lysates of Vero cells infected with DENV-2 and 293T cells transfected with the DENV-2-C expression plasmid encoding Flag were used as antigens. Their reactivity against various antibodies was analyzed by Western blotting. Anti-Flag antibody was used as a positive control of capsid protein expression. Arrowheads showed 12 kDa. (B) Indirect immunofluorescence assay of monoclonal antibodies using Vero cells transfected with the DENV-2 capsid protein expression plasmid and fixed 48 hours following infection with $4 \%$ paraformaldehyde.

Note: Cells were stained with each monoclonal antibody and anti-Flag antibody, followed by Alexa Fluor 488 goat anti-mouse lgG secondary antibody.

phylogenetically to that of DENV-4, but very far from those of DENV-1 and DENV-3. On the other hand, phylogenies of the envelope and nonstructural 1 proteins showed different branching orders among the four serotypes, with DENV-4 being the first diverge, followed by DENV-2, and the final diverge being between DENV-1 and DENV-3 (Figure 4, envelope and nonstructural 1 proteins). The distances between DENV clusters seem to be comparable with those between Japanese encephalitis virus and West Nile virus (Figure 4). Furthermore, clusters in the flavivirus capsid protein showed more diversity than those in the flavivirus envelope and nonstructural 1 proteins (Figure 4).

\section{Discussion}

In this study, we produced mouse monoclonal antibodies to the capsid protein of DENV. Five hybridoma cells producing anti-capsid monoclonal antibodies were successfully generated and characterized. Interestingly, none of the antibodies generated could bind with all serotypes of DENV. Consequently, this result was greatly different from our initial expectations. Phylogenetic tree analysis using databasederived sequences of DENV-1 to DENV-4 supported the above result, because capsid protein variations were higher than our initial expectation, as confirmed by comparison with other viral proteins, such as the envelope and nonstructural 1 proteins.

By immunization of BALB/c mice with DENV-2 antigens, we obtained only five monoclonal antibodies reactive with DENV capsid protein among the 60 monoclonal antibodies. Similarly, it has been reported that only a few antiDENV capsid protein monoclonal antibodies are obtained by immunization with the whole virus. ${ }^{32}$ In this study, the C2 monoclonal antibody reacted with DENV capsid protein by both Western blotting and immunofluorescence assays,

Table I Summary of cross-reactivity of MAb within Flavivirus and specificity MAb against DENV-2-C protein

\begin{tabular}{|c|c|c|c|c|c|c|}
\hline & DENV-I* & DENV-2 & DENV-3 & DENV-4 & JEV & DENV-2-C plasmid** \\
\hline 4G2 & + & + & + & + & + & - \\
\hline $\mathrm{C} 2$ & - & + & - & - & - & + \\
\hline C99 & - & + & - & + & - & + \\
\hline $5 F I 2$ & - & + & - & - & - & + \\
\hline 5D9 & - & + & - & - & - & + \\
\hline 5DI2 & - & + & - & + & - & + \\
\hline Anti-Flag & ND & ND & ND & ND & ND & + \\
\hline
\end{tabular}

Notes: *DENV-I to -4 and JEV infected Vero cell; **DENV2-C protein expression plasmid transfected Vero.

Abbreviations: +, positive; -, negative; ND, not done. 


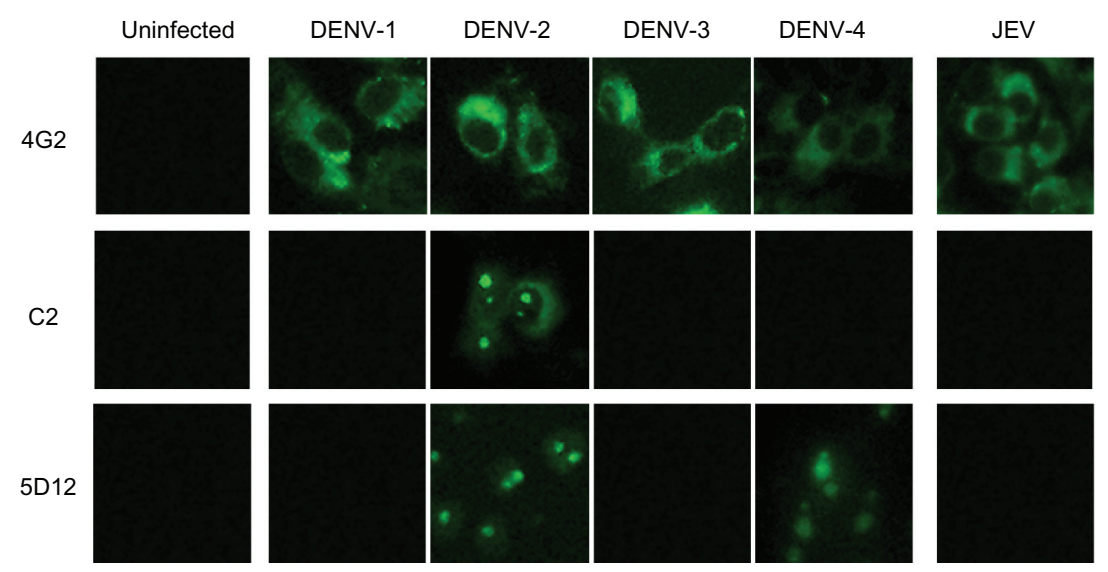

Figure 3 Determination of cross-reactivities of monoclonal antibodies to flavivirus.

Notes: Vero cells, infected with each DENV serotype (I to 4) and Japanese encephalitis virus, or uninfected Vero cells were stained using the indicated monoclonal antibodies with Alexa Fluor ${ }^{\circledR} 488$ goat anti-mouse IgG secondary antibody. The cross-reactivities of other monoclonal antibodies are summarized in Table I.

while the C99, 5F12, 5D9, and 5D12 monoclonal antibodies reacted with the capsid protein only on immunofluorescence assay. These results suggest that the $\mathrm{C} 2$ monoclonal antibody recognized a linear epitope, and other monoclonal antibodies recognized conformational epitopes, and the structure of these conformation epitopes might be lost by cell-lysis buffer treatment. Further work is needed on the structural analysis of this epitope.

Three monoclonal antibodies were specific to DENV-2 and the other two clones were cross-reactive with DENV-2 and DENV-4. Previous research on anti-DENV capsid protein has reported similar results for serotype specificity and/or low cross-reactivity with DENV capsid protein. ${ }^{6,32-34}$ However, the reason for the low cross-reactivity of DENV capsid protein has not been discussed in these papers. Our bioinformatics characterization of DENV capsid protein sequences from the database revealed that DENV-2 and DENV-4 were clustered in the same branch, while DENV-1 and DENV-3 clustered in the other branch (Figure 4). However, these classifications of DENV capsid protein were not correlated with those of the DENV envelope and nonstructural 1 proteins. In general, epitopes recognized with antibodies are short amino acid

Table 2 Number of amino acid sequences of Flavivirus extracted from NCBI database

\begin{tabular}{llll}
\hline & Capsid & Envelope & NSI \\
\hline DENV-I & 116 & 805 & 275 \\
DENV-2 & 101 & 689 & 242 \\
DENV-3 & 90 & 391 & 181 \\
DENV-4 & 32 & 217 & 44 \\
WNV & 64 & 245 & 124 \\
JEV & 60 & 353 & 65 \\
\hline
\end{tabular}

Abbreviations: DENV, Dengue virus; WNV, West nile virus; JEV, Japanese encephalitis virus. sequences or protein conformations; therefore, it may be difficult to discuss serological cross-reactivity using only phylogenetic trees from amino acid sequences. However, our bioinformatics characterization results for the capsid protein may correlate well with the results for anti-capsid monoclonal antibodies obtained by immunization with DENV-2 antigens
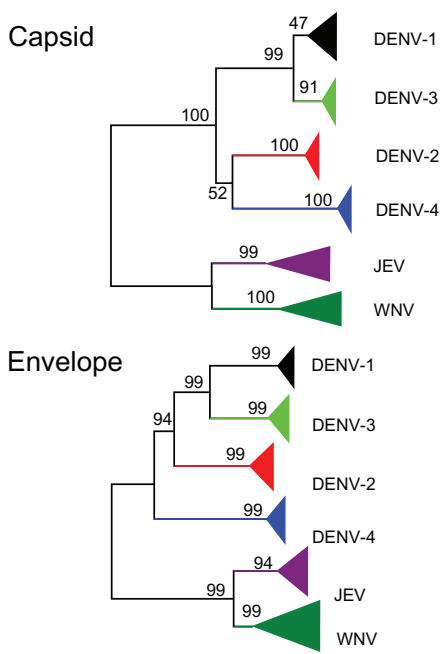

NS1

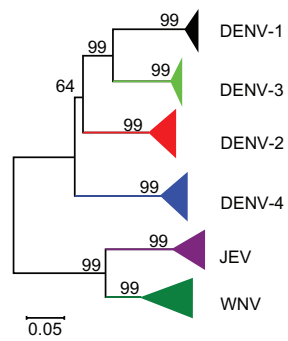

Figure 4 Phylogenetic trees of flavivirus capsid, envelope, and nonstructural I protein inferred using the neighbor-joining method.

Notes: All positions containing gaps and missing data were eliminated. Bootstrap values obtained from 500 replicates were shown on major branches. DENV-I to DENV-4 strains are shown in black, red, light green, and blue, respectively. West Nile and Japanese encephalitis viruses are shown in dark green, and purple, respectively. 
cross-reactive with DENV-2 and DENV-4, but not with those for DENV-1 and/or DENV-3. The phylogenetic tree for the flavivirus capsid proteins also showed that the distance between DENV-2 and DENV-4 clusters and between DENV-1 and DENV-3 clusters was comparable with that between Japanese encephalitis virus and West Nile virus. This bioinformatics information suggests that DENV-2 capsid antibodies that are cross-reactive with DENV-4 are relatively frequent, whereas those that are cross-reactive with DENV-1 and/or DENV-3 are rare (Table 1). The diversity between DENV serotypes was comparable with those between Japanese encephalitis virus and West Nile virus (Figure 4). These tendencies were correlated with those not only in capsid proteins, but also in other proteins such as envelope and nonstructural 1 proteins. Our results show that phylogenetic distances between the four serotypes of DENV were as different as that of Japanese encephalitis virus and West Nile virus. However, the diversity of flavivirus capsid protein was much higher than that for envelope and nonstructural 1 proteins. Therefore, these data suggest that there may be difficulty in establishing monoclonal antibodies against DENV capsid protein that are cross-reactive with all the subtypes of DENV. The monoclonal antibodies specific for DENV-2 generated in this study could be used for serotyping of DENV infection. However, DENV capsid protein can be used for serotyping of DENV infection but not for rapid diagnostic test kits to detect all four DENV serotypes.

The levels of diversity among the DENV serotypes reflect the differences between species of flavivirus. This high diversity of DENV correlates with the classification of individual DENV serotypes. However, it is well known that there is significant diversity among genotypes for the individual DENV serotypes. ${ }^{35,36}$ These results suggest that individual DENV serotypes have evolved in their own particular way, unlike other flaviviruses.

In this study, the monoclonal antibodies generated specifically for DENV-2 capsid protein can be used for serotyping of DENV infection and for early diagnosis of high titers of viremia in patients with hemorrhagic Dengue fever or Dengue shock syndrome caused by DENV-2 infection. We could not obtain monoclonal antibodies cross-reactive with all four serotypes using mice immunized with a sole serotype of DENV. Our phylogenetic analysis of DENV and the high diversity of the DENV capsid protein sequence support this conclusion.

\section{Acknowledgments}

We are grateful to Pratap Singhasivanon (Faculty of Tropical Medicine, Mahidol University) and Shigeyuki
Hamada (Research Collaboration Center on Emerging and Re-emerging Infections, Research Institute for Microbial Diseases, Osaka University) for their valuable help with this study. The manuscript was proofread by the Medical English Service (Kyoto, Japan). This work was supported by the Japan Initiative for Global Research Network on Infectious Diseases program managed by the Ministry of Education, Cultures, Sports, Science, and Technology of Japan.

\section{Disclosure}

The authors report no conflicts of interest in this work.

\section{References}

1. Gubler DJ. Epidemic dengue/dengue hemorrhagic fever as a public health, social and economic problem in the 21 st century. Trends Microbiol. 2002;10(2):100-103.

2. Mackenzie JS, Gubler DJ, Petersen LR. Emerging flaviviruses: the spread and resurgence of Japanese encephalitis, West Nile and dengue viruses. Nat Med. 2004;10(Suppl 12):S98-S109.

3. Lindenbach DR, Rice CM. Flaviviridae: The Viruses and their Replication. Philadelphia, PA: Lippincott Williams \& Wilkins; 2001:4.

4. Kuhn RJ, Zhang W, Rossmann MG, et al. Structure of dengue virus: implications for flavivirus organization, maturation, and fusion. Cell. 2002;108(5):717-725.

5. Sangiambut S, Keelapang P, Aaskov J, et al. Multiple regions in dengue virus capsid protein contribute to nuclear localization during virus infection. J Gen Virol. 2008;89(Pt 5):1254-1264.

6. Bulich R, Aaskov JG. Nuclear localization of dengue 2 virus core protein detected with monoclonal antibodies. J Gen Virol. 1992;73(Pt 11): 2999-3003.

7. Keelapang P, Sriburi R, Supasa S, et al. Alterations of pr-M cleavage and virus export in pr-M junction chimeric dengue viruses. $J$ Virol. 2004;78(5):2367-2381.

8. Andersen JS, Lam YW, Leung AK, et al. Nucleolar proteome dynamics. Nature. 2005;433(7021):77-83.

9. Boisvert FM, van Koningsbruggen S, Navascues J, Lamond AI. The multifunctional nucleolus. Nat Rev Mol Cell Biol. 2007;8(7):574-585.

10. Oh W, Yang MR, Lee EW, et al. Jab1 mediates cytoplasmic localization and degradation of West Nile virus capsid protein. J Biol Chem. 2006;281(40):30166-30174.

11. Mori Y, Okabayashi T, Yamashita T, et al. Nuclear localization of Japanese encephalitis virus core protein enhances viral replication. J Virol. 2005;79(6):3448-3458.

12. Moriishi K, Okabayashi T, Nakai K, et al. Proteasome activator PA28 gamma-dependent nuclear retention and degradation of hepatitis $\mathrm{C}$ virus core protein. J Virol. 2003;77(19):10237-10249.

13. Westaway EG, Khromykh AA, Kenney MT, Mackenzie JM, Jones MK. Proteins C and NS4B of the flavivirus Kunjin translocate independently into the nucleus. Virology. 1997;234(1):31-41.

14. Munene E, Songok E, Nyamongo JA, Langat DK, Otsyula M. Evaluation of HIV ELISA diagnostic kit developed at the Institute of Primate Research, Nairobi, Kenya. Afr J Health Sci. 2002;9(3-4):117-122.

15. Chomel JJ, Thouvenot D, Onno M, Kaiser C, Gourreau JM, Aymard M. Rapid diagnosis of influenza infection of NP antigen using an immunocapture ELISA test. J Virol Methods. 1989;25(1):81-91.

16. Otteken A, Nick S, Bergter W, et al. Identification of a gag protein epitope conserved among all four groups of primate immunodeficiency viruses by using monoclonal antibodies. J Gen Virol. 1992;73(Pt 10):2721-2724.

17. Minassian AA, Kalyanaraman VS, Gallo RC, Popovic M. Monoclonal antibodies against human immunodeficiency virus (HIV) type 2 core proteins: cross-reactivity with HIV type 1 and simian immunodeficiency virus. Proc Natl Acad Sci U S A. 1988;85(18):6939-6943. 
18. Zaki A, Coudrier D, Yousef AI, Fakeeh M, Bouloy M, Billecocq A. Production of monoclonal antibodies against Rift Valley fever virus: application for rapid diagnosis tests (virus detection and ELISA) in human sera. J Virol Methods. 2006;131(1):34-40.

19. Kimura T, Rokuhara A, Matsumoto A, et al. New enzyme immunoassay for detection of hepatitis B virus core antigen ( $\mathrm{HBcAg}$ ) and relation between levels of HBcAg and HBV DNA. J Clin Microbiol. 2003;41(5):1901-1906.

20. Fabrizi F, Bromberg J, Elli A, Dixit V, Martin P. Review article: hepatitis $\mathrm{C}$ virus and calcineurin inhibition after renal transplantation. Aliment Pharmacol Ther. 2005;22(8):657-666.

21. Saijo M, Niikura M, Ikegami T, Kurane I, Kurata T, Morikawa S. Laboratory diagnostic systems for Ebola and Marburg hemorrhagic fevers developed with recombinant proteins. Clin Vaccine Immunol. 2006;13(4):444-451.

22. Dussart P, Labeau B, Lagathu G, et al. Evaluation of an enzyme immunoassay for detection of dengue virus NS1 antigen in human serum. Clin Vaccine Immunol. 2006;13(11):1185-1189.

23. Kumarasamy V, Wahab AH, Chua SK, et al. Evaluation of a commercial dengue NS1 antigen-capture ELISA for laboratory diagnosis of acute dengue virus infection. J Virol Methods. 2007;140(1-2):75-79.

24. Kanai Y, Chittaganpitch M, Nakamura I, et al. Distinct propagation efficiencies of H5N1 influenza virus Thai isolates in newly established murine respiratory region-derived cell clones. Virus Res. 2010;153(2):218-225.

25. Kurosu T, Khamlert C, Phanthanawiboon S, Ikuta K, Anantapreecha S. Highly efficient rescue of dengue virus using a co-culture system with mosquito/mammalian cells. Biochem Biophys Res Commun. 2010;394(2):398-404.

26. Masrinoul P, Diata MO, Pambudi S, Limkittikul K, Ikuta K, Kurosu T. Highly conserved region 141168 of the NS1 protein is a new common epitope region of dengue virus. Jpn J Infect Dis. 2011;64(2):109-115.

27. Altschul SF, Madden TL, Schaffer AA, et al. Gapped BLAST and PSI-BLAST: a new generation of protein database search programs. Nucleic Acids Res. 1997;25(17):3389-3402.
28. Katoh K, Toh H. Improved accuracy of multiple ncRNA alignment by incorporating structural information into a MAFFT-based framework. BMC Bioinformatics. 2008;9:212.

29. Saitou N, Nei M. The neighbor-joining method: a new method for reconstructing phylogenetic trees. Mol Biol Evol. 1987;4(4):406-425.

30. Tamura K, Peterson D, Peterson N, Stecher G, Nei M, Kumar S. MEGA5: molecular evolutionary genetics analysis using maximum likelihood, evolutionary distance, and maximum parsimony methods. Mol Biol Evol. 2011;28(10):2731-2739.

31. Falconar AK. Identification of an epitope on the dengue virus membrane $(\mathrm{M})$ protein defined by cross-protective monoclonal antibodies: design of an improved epitope sequence based on common determinants present in both envelope (E and M) proteins. Arch Virol. 1999; 144(12):2313-2330.

32. Puttikhunt C, Ong-Ajchaowlerd P, Prommool T, et al. Production and characterization of anti-dengue capsid antibodies suggesting the $\mathrm{N}$ terminus region covering the first 20 amino acids of dengue virus capsid protein is predominantly immunogenic in mice. Arch Virol. 2009;154(8):1211-1221.

33. Tadano M, Makino Y, Fukunaga T, Okuno Y, Fukai K. Detection of dengue 4 virus core protein in the nucleus. I. A monoclonal antibody to dengue 4 virus reacts with the antigen in the nucleus and cytoplasm. J Gen Virol. 1989;70(Pt 6):1409-1415.

34. Gagnon SJ, Zeng W, Kurane I, Ennis FA. Identification of two epitopes on the dengue 4 virus capsid protein recognized by a serotypespecific and a panel of serotype-cross-reactive human CD4+ cytotoxic T-lymphocyte clones. J Virol. 1996;70(1):141-147.

35. Rico-Hesse R. Molecular evolution and distribution of dengue viruses type 1 and 2 in nature. Virology. 1990;174(2):479-493.

36. Holmes EC, Twiddy SS. The origin, emergence and evolutionary genetics of dengue virus. Infect Genet Evol. 2003;3(1):19-28.
Biologics: Targets \& Therapy

\section{Publish your work in this journal}

Biologics: Targets \& Therapy is an international, peer-reviewed journal focusing on the patho-physiological rationale for and clinical application of Biologic agents in the management of autoimmune diseases, cancers or other pathologies where a molecular target can be identified. This journal is indexed on PubMed Central, CAS, EMBase, Scopus

\section{Dovepress}

and the Elsevier Bibliographic databases. The manuscript management system is completely online and includes a very quick and fair peerreview system, which is all easy to use. Visit http://www.dovepress com/testimonials.php to read real quotes from published authors. 